Reply to Comment on Saku et al. : Reasons and risk factors for ninety day re-admission following primary total knee arthroplasty in a high-volume centre

Saku, Sami A.

2018-06

Saku , S A , Madanat , R \& Mäkinen , T J 2018 , ' Reply to Comment on Saku et al. Reasons and risk factors for ninety day re-admission following primary total knee arthroplasty in a high-volume centre ' , International Orthopaedics , vol. 42 , no. 6 , pp. 1423-1424 . https://doi.org/10.1007/s00264-01

http://hdl.handle.net/10138/302402

https://doi.org/10.1007/s00264-018-3923-x

publishedVersion

Downloaded from Helda, University of Helsinki institutional repository.

This is an electronic reprint of the original article.

This reprint may differ from the original in pagination and typographic detail.

Please cite the original version. 


\title{
Reply to Comment on Saku et al.: Reasons and risk factors for ninety day re-admission following primary total knee arthroplasty in a high-volume centre
}

\author{
Sami A. Saku ${ }^{1}$ (D) $\cdot$ Rami Madanat ${ }^{1} \cdot$ Tatu J. Mäkinen ${ }^{1}$ \\ Received: 21 March 2018 / Accepted: 26 March 2018 / Published online: 3 April 2018 \\ (C) SICOT aisbl 2018
}

Dear Editor,

We thank Dr. Alves and Dr. Kendall for their interest in our paper and for their questions that highlight some need for further clarification in our article entitled "Reasons and risk factors for ninety day re-admission following primary total knee arthroplasty in a high-volume centre" [1].

Dr. Alves and Dr. Kendall highlight a relevant topic about overfitting of statistical models. In our study, we did account for this possible problem by choosing an appropriate statistical method for the risk factor analysis. First, we used univariate logistic regression to investigate associations between potential risk factors and re-admission. In the second step, we included 13 risk factors with $P$ values $<0.10$ in the univariate analysis into the stepwise logistic regression. We used stepwise logistic regression to reduce the number of predictors in the final multivariable model and included four predictors in the final multivariable model. In this final model, there were 30 re-admissions per predictor variable. The usual recommendation for event/number-of-predictors ratio is at least 10 to avoid overfitting [2]. Therefore, we believe that we did not have any problems with overfitting the model.

Secondly, we did not include any analysis of post-operative analgesic strategies as all patients are primarily treated by the same multimodal analgesic strategy. At the time of the study, the pain management strategy consisted of non-steroidal anti-inflammatory drugs, acetaminophen and opioid analge-

Sami A. Saku

sami.saku@helsinki.fi

1 Department of Orthopedics and Traumatology, University of Helsinki and Helsinki University Hospital, PL 900, Sairaalakatu 1, 00029 HUS Vantaa, Finland sics. According to our discharge criteria, the patients are only discharged when the pain is manageable with oral pain killers.

There are surely minor interpatient differences in the amount of peri-operative short-acting analgesics consumed, but we find it very unlikely that this could have any significant effect on the number of re-admissions due to pain. As it is well-known, and as we have also shown in a recent study at our institution [3], pain is an unfortunately common issue after total knee arthroplasty. It seems plausible though that pain solely caused by under- or misuse of analgesics does not generally lead to re-admissions. Before being re-admitted to the ward, the patients are carefully evaluated and their pain medication adjusted both when contacting the hospital by phone and when visiting the emergency department.

Lastly, we agree with Dr. Alves and Dr. Kendall that education and health literacy might be linked to hospital re-admissions in some clinical settings. Though in the study that Dr. Alves and Dr. Kendall quoted [4], the authors did not find health literacy to be a statistically significant risk factor for re-admission.

To ensure that every patient receives and understands the instructions for post-operative care at our institution, every patient receives both written and oral instructions, both at the pre-operative visit and prior to discharge after the surgery. The patients are not discharged until the nursing staff are assured that the patients have clearly understood the instructions for post-operative rehabilitation, wound management etc. using standardized discharge criteria. Furthermore, all patients are encouraged to attend our patient education lectures given on a regular basis.

As the most common reason for re-admission in our study was periprosthetic infection, and most re-admissions were strictly due to medical or surgical issues, it does not seem likely that health literacy is a substantial confounder in our study.

We hope that the above clarifications will help with further validating the findings of our study. 
Funding This study was supported by grants from Svenska Kulturfonden and Ålands Kulturstiftelse r.f.

\section{Compliance with ethical standards}

Conflict of interest The authors declare that they have no conflict of interest.

\section{References}

1. Saku SA, Madanat R, Mäkinen TJ (2018) Reasons and risk factors for ninety day re-admission following primary total knee arthroplasty in a high-volume Centre. Int Orthop 42(1):95-99. https://doi.org/10. 1007/s00264-017-3676-y

2. Vittinghoff E, McCulloch CE (2007) Relaxing the rule of ten events per variable in logistic and cox regression. Am J Epidemiol 165(6): 710-718. https://doi.org/10.1093/aje/kwk052

3. Hällfors E, Saku SA, Mäkinen TJ, Madanat R (2018) A consultation phone service for patients with total joint arthroplasty may reduce unnecessary emergency department visits. J Arthroplast 33:650-654. https://doi.org/10.1016/j.arth.2017.10.040

4. Mitchell SE, Sadikova E, Jack BW, Paasche-Orlow MK (2012) Health literacy and 30-day postdischarge hospital utilization. J Health Commun 17(SUPPL. 3):325-338. https://doi.org/10.1080/ 10810730.2012 .715233 